\title{
PREditOR: a synthetic biology approach to removing heterochromatin from cells
}

\author{
Oscar Molina (D) - Mar Carmena - Isabella E. Maudlin • \\ William C. Earnshaw
}

Received: 11 November 2016/Revised: 13 November 2016 / Accepted: 16 November 2016 / Published online: 6 December 2016

(C) The Author(s) 2016. This article is published with open access at Springerlink.com

\begin{abstract}
It is widely accepted that heterochromatin is necessary to maintain genomic stability. However, direct experimental evidence supporting this is slim. Previous studies using either enzyme inhibitors, gene knockout or knockdown studies all are subject to the caveat that drugs may have off-target effects and enzymes that modify chromatin proteins to support heterochromatin formation may also have numerous other cellular targets as well. Here, we describe PREditOR (protein reading and editing of residues), a synthetic biology approach that allows us to directly remove heterochromatin from cells without either drugs or global interference with gene function. We find that removal of heterochromatin perturbs mitotic progression and causes a dramatic increase in chromosome segregation defects, possibly as a result of interfering with the normal centromeric localization of the chromosomal passenger complex.
\end{abstract}

Keywords Heterochromatin - Chromosome segregation $\cdot$ Centromeres $\cdot$ Mitosis

Responsible Editor: Conly Rieder.

Electronic supplementary material The online version of this article (doi:10.1007/s10577-016-9539-3) contains supplementary material, which is available to authorized users.

O. Molina $(\bowtie) \cdot$ M. Carmena · I. E. Maudlin •

W. C. Earnshaw $(\bowtie)$

Wellcome Trust Centre for Cell Biology, University of Edinburgh,

Edinburgh EH9 3BF, UK

e-mail: oscar.molina@ed.ac.uk

e-mail: bill.earnshaw@ed.ac.uk

\section{Introduction}

Eukaryotic genomes are organized as a spectrum of global chromatin states with differing epigenetic profiles. The first of these to be identified in interphase nuclei as regions of compacted and decompacted chromatin were termed heterochromatin and euchromatin (Bannister and Kouzarides 2011). Heterochromatin is a transcriptionally repressive chromatin state that can be either facultative or constitutive (Oberdoerffer and Sinclair 2007). The former is a transient epigenetic state found at promoters that changes in response to the environment and during development to establish tissue-specific gene expression and differentiation. Constitutive heterochromatin remains compacted permanently throughout cell differentiation and in different cell types. Most constitutive heterochromatin is found at pericentromeric regions, although it can also be found at other sites, including telomeres (Saksouk et al. 2015) and the long arm of the $\mathrm{Y}$ chromosome in mammals.

Centromeres, defined cytologically as the primary constriction of mitotic chromosomes, are the loci that direct chromosome segregation during cell division (Fukagawa and Earnshaw 2014). Human centromeres contain long stretches of non-coding alpha-satellite DNA organized in high-order repeats (Aldrup-Macdonald and Sullivan 2014). Centromeres can be divided into two major compartments, the core centromere of "centrochromatin" (Sullivan and Karpen 2004) and the pericentromere. Centrochromatin nucleates the assembly of the kinetochore, a multi-protein complex that binds microtubules and directs chromosome segregation 
(Fukagawa and Earnshaw 2014). Centrochromatin is characterized by the presence of nucleosomes containing the centromere-specific histone H3 CENP-A (Earnshaw and Migeon 1985) interspersed with nucleosomes containing canonical histone $\mathrm{H} 3$ bearing epigenetic modifications associated with transcriptionally active chromatin, such as H3K4me2 and H3K36me2 (Sullivan and Karpen 2004; Bergmann et al. 2011; Fukagawa and Earnshaw 2014). Centrochromatin is flanked by pericentromeric heterochromatin distinguished by the presence of characteristic histone modifications, including histone $\mathrm{H} 3$ trimethylated on lysine 9 (H3K9me3), which binds heterochromatin protein 1 (HP1) and histone H4 trimethylated on lysine 20 (H4K20me3) (Allshire et al. 1995; Ekwall et al. 1995; Bannister et al. 2001).

Diverse functional roles have been attributed to pericentromeric heterochromatin. These include facilitating sister chromatid cohesion by recruiting and retaining cohesin complexes in metaphase (Bernard et al. 2001; Nonaka et al. 2002; Yamagishi et al. 2008; Gartenberg 2009), suppressing inappropriate homologous recombination between repetitive DNA elements (Peng and Karpen 2007) and kinetochore maintenance. Indeed, euchromatin-heterochromatin boundaries have been suggested to be preferred sites for kinetochore formation in yeast and Drosophila melanogaster (Folco et al. 2008; Olszak et al. 2011).

Despite disagreements regarding its functions (Bernard et al. 2001; Koch et al. 2008), the importance of proper regulation and maintenance of pericentromeric heterochromatin is suggested by the finding that knockout mice for the H3K9-specific methyltransferase Suv39h1 show increased chromosomal instability (CIN), embryonic lethality and are prone to tumour formation (Peters et al. 2001). Furthermore, cancer cells with lower levels of pericentromeric heterochromatin show CIN (Slee et al. 2012). However, the precise role of heterochromatin in regulating proper chromosome segregation remains unclear.

Epigenetic regulation of chromatin can be described as a series of consecutive steps in which an EDITOR (writer or eraser) makes or removes a MARK on a chromatin protein. A READER can either recognize this MARK or cease to do so if the mark is removed. The binding of the READER establishes a CHROMATIN STATE, such as euchromatin, heterochromatin or centrochromatin. We refer to this as an $\mathrm{E} \rightarrow$ $\mathrm{M} \rightarrow \mathrm{R} \rightarrow \mathrm{C}$ pathway. Here, we present a novel
Fig. 1 Tethering JMJD2D to heterochromatin decrease H3K9me3 levels. a Schematic of the PREdiTOR approach to tether chromatin modifiers to heterochromatin regions. b Schematic drawings of the SUV39H1 $\triangle$ SET-EYFP fusion constructs. c Diagram of the experimental design. d Representative immunofluorescence images of HeLa cells expressing the indicated SUV39H1 $\triangle$ SET-EYFP fusion proteins and stained for H3K9me3. Scale bar $10 \mu \mathrm{m}$. e Quantification of fluorescence signals of $\mathrm{H} 3 \mathrm{~K} 9 \mathrm{me} 3$ staining in individual transfected cells as in d plotted as arbitrary fluorescence units (A.F.U). Solid bars indicate the medians of three independent experiments and error bars represent the standard error of the mean (s.e.m). Asterisks indicate statistical significant differences compared to EYFP $(* P<0.05 ; * * P<0.001$; Student's $t$ test $)$

synthetic biology approach called PREditOR (protein reading and editing of residues) to dissect and manipulate $\mathrm{E} \rightarrow \mathrm{M} \rightarrow \mathrm{R} \rightarrow \mathrm{C}$ pathways and analyse their functional outcome.

In order to study the role(s) of heterochromatin on chromosome segregation, we designed a PREditOR strategy that allows us to remove heterochromatin without drug treatments or global protein knockdowns. Our studies reveal that heterochromatin removal from pericentromeric regions leads to chromosome segregation defects as a result of disruption of kinetochore structure, chromosome passenger complex delocalization and decreases in centromeric stiffness during mitosis.

\section{Material and methods}

\section{Expression constructs}

The SUV39H1 $\Delta$ SET-EYFP constructs were obtained as follows. The chromodomain of SUV39H1 was amplified from a custom made cDNA library from HeLa cells and cloned into the NheI and AgeI restriction sites of the pYIP-EYFP vector (Bergmann et al., 2011) generating SUV39H1 $\triangle$ SET-EYFP, which contains $3^{\prime}$ attL and attR sites for Gateway cloning (Fisher Technologies). Full length JMJD2D was PCR amplified from our cDNA library using the oligonucleotides JMJD-Fw (5'caccatggaaactatgaagtc $-3^{\prime}$ ) and JMJD-Rv (5'ttaaacgggcacagg- $\left.3^{\prime}\right)$. The PCR product was used for gateway cloning following the manufacturer's instructions (Fisher Technologies), to generate the construct pYIP-SUV39H1 $\Delta$ SET-EYFP-JMJD2D ${ }^{\text {WT }}$, which express this fusion protein from a CMV promoter and confers resistance to puromycin. To generate the D195A 

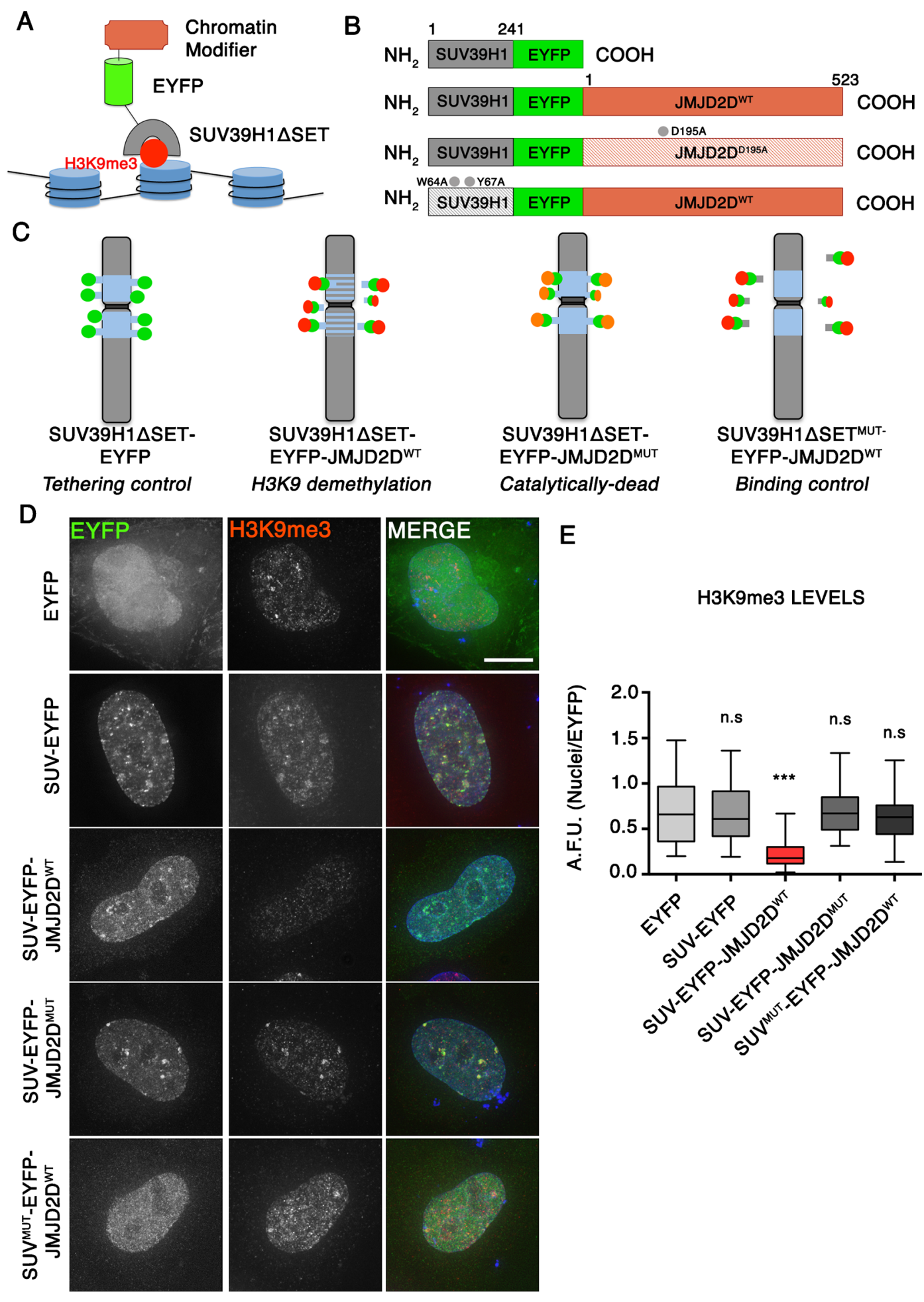

mutant of JMJD2D and the W64AY67A double mutant of SUV39H1 $\Delta$ SET, the pYIP-SUV39H1 $\Delta$ SET-EYFP-
JMJD2D ${ }^{\text {WT }}$ construct was subjected to site-directed mutagenesis using the QuikChange II kit (Stratagene). 

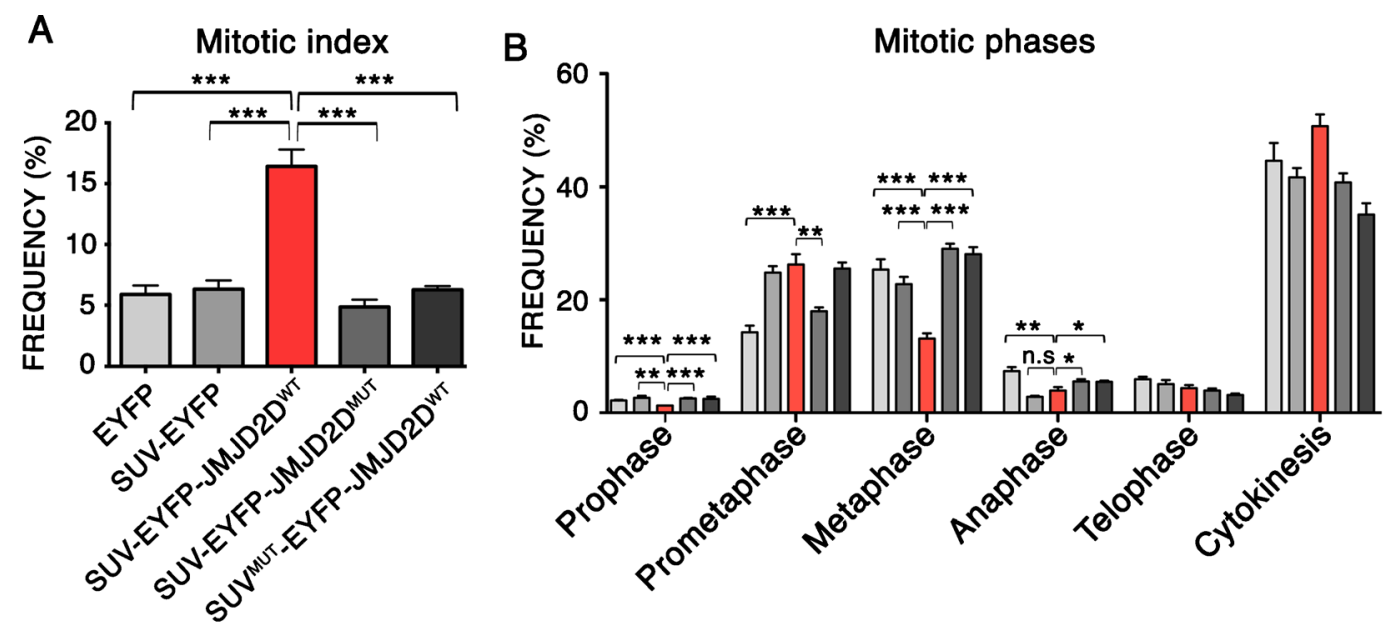

C

D Abnormal mitoses $E$ Bridges and lagging chr
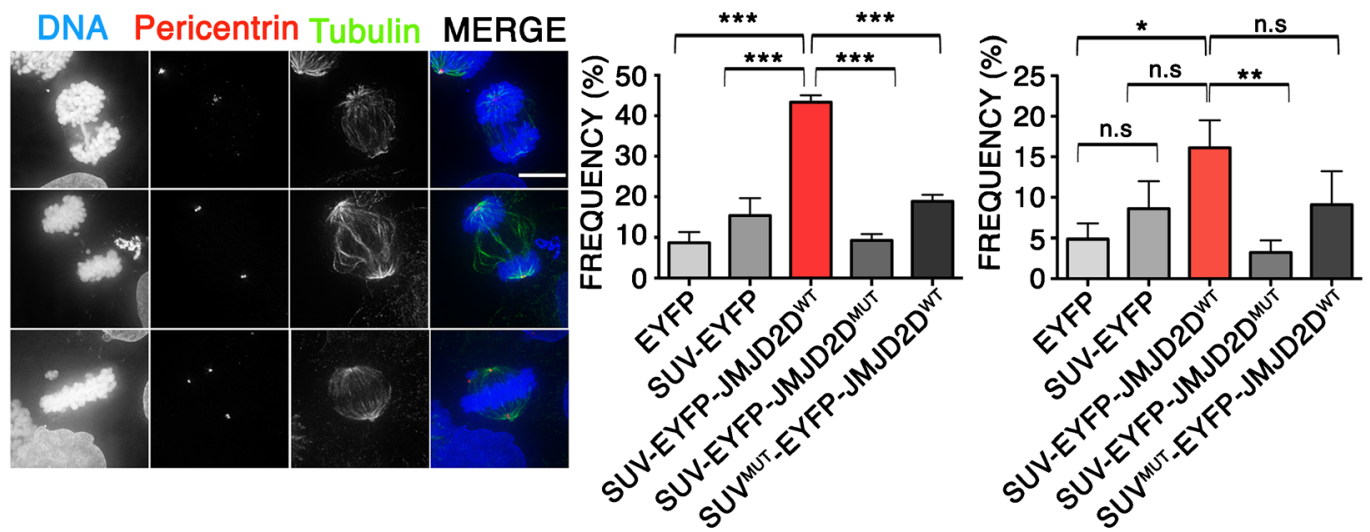

F

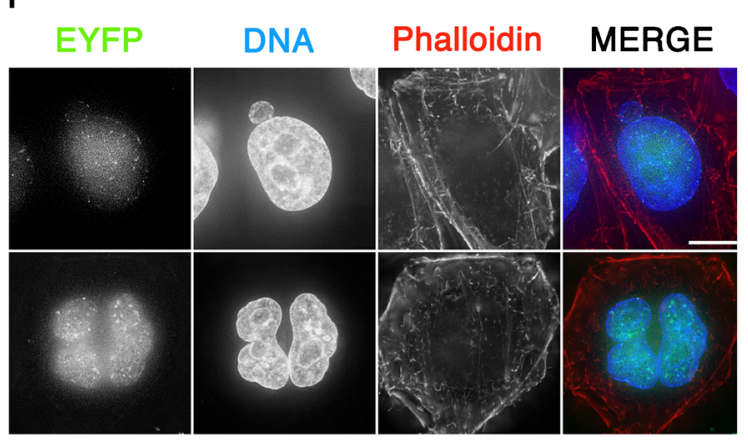

G

Interphase abnormalities

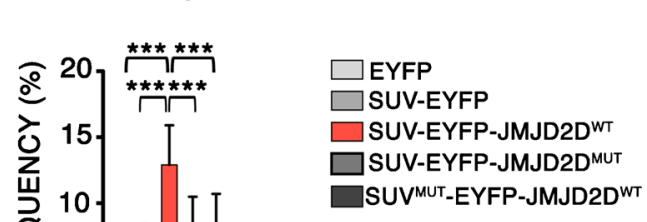

Cell culture, transfections and drug treatments

HeLa cells were maintained in DMEM supplemented with 5\% FBS (Invitrogen), $100 \mathrm{U} / \mathrm{ml}$ penicillin $\mathrm{G}$ and $100 \mu \mathrm{g} / \mathrm{ml}$ streptomycin sulphate (Invitrogen). Cells were grown at $37{ }^{\circ} \mathrm{C}$ in $5 \% \mathrm{CO}_{2}$ in a humidified atmosphere. Transfections were performed using Xtremegene-9 (Roche) following the manufacturer's instructions. In brief, for transfections of cells growing in 24-well plates on polylysine-coated glass coverslips, transfection 
4Fig. 2 Heterochromatin removal disrupts mitosis and chromosome segregation. a Analysis of the frequency of mitotic cells after expressing the indicated SUV39H1 $\triangle$ SET-EYFP fusion proteins. Data represent the mean and standard error of the mean (s.e.m) of five independent experiments. b Analysis of the frequency of every individual mitotic phase in relation of the total number of mitoses. Data represents the mean and the standard error of the mean (s.e.m) of six independent experiments. c Representative IF images showing mitotic abnormalities in HeLa cells. Images show examples of chromosome bridges (top), lagging chromosomes (middle) and uncongressed chromosomes (bottom). d Analysis of the frequency of abnormal mitoses after expressing the indicated SUV39H1 $\triangle$ SET-EYFP fusion proteins. Data represent the mean and standard error of the mean (s.e.m) of four independent experiments. e Analysis of the frequency of mitotic cells showing bridges or lagging chromosomes after expression of the indicated SUV39H1 $\triangle$ SET-EYFP fusion proteins. Data represent the mean and standard error of the mean (s.e.m) of four independent experiments. f Representative IF images showing interphase abnormalities in HeLa cells. Images show a cell with micronucleus (top), and a binucleate cell (bottom). g Quantification of interphase abnormalities after expressing the indicated SUV39H1 $\triangle$ SET-EYFP fusion proteins. Data represent the mean and standard error of the mean (s.e.m) of three independent experiments. Asterisks indicate statistical significant differences compared to EYFP $(* P<0.05$, $* * P<0.01$, $* * * P<0.001 ;$ Student's $t$ test)

complexes containing $3 \mu \mathrm{l}$ Xtremegene-9 reagent and $1 \mu \mathrm{g}$ plasmid DNA were prepared in $100 \mu \mathrm{l}$ OptiMEM (Invitrogen). After $20 \mathrm{~min}$ of incubation at room temperature, $25 \mu \mathrm{l}$ of transfection complexes was added dropwise in each well. After $24 \mathrm{~h}$, transfected cells were selected adding $2 \mu \mathrm{g} / \mathrm{ml}$ of Puromycin (Sigma) and grown for 24 additional hours before fixation.

For RNAi treatments, HeLa cell transfections were performed using Polyplus jetPRIME (PEQLAB, Southampton, UK) with the indicated amounts of siRNA oligos and $500 \mathrm{ng}$ of plasmid DNA. After $24 \mathrm{~h}$, fresh DMEM was added and cells were maintained for 24 additional hours before fixation.

Indirect immunofluorescence and microscopy

Indirect immunofluorescence staining of cells fixed in 2.6 to $4 \%$ Formaldehyde/1xPBS was performed following standard procedures. The following antibodies were used: rabbit anti-H3K9me3 (abcam ab8898, 3\% formaldehyde, 1/500), mouse anti-HP1 $\alpha$ (Millipore MAB3584, 2.6\% Formaldehyde, 1/1000), rabbit anti-CENP-C (R554, $2.6 \%$ formaldehyde, $1 / 500$ ), rabbit anti-CENP-B (WCEB4, 2.6\% formaldehyde, 1/500), mouse anti-
HEC1 (abcam AC3612, $2.6 \%$ formaldehyde, 1/1000), mouse anti-Tubulin (Sigma B516, 1/2000), rabbit antiSGO1 (A. Losada, 4\% formaldehyde, 1/1000), rabbit anti-SMC2 (A. Losada, 4\% formaldehyde, 1/1000), rabbit anti-Pericentrin (abcam AB4448; 4\% formaldehyde, 1/500), rabbit anti-Survivin (Cell Signalling, 4\% formaldehyde, 1/400) and rabbit anti-INCENP (Cell Signalling P240, 4\% formaldehyde, 1/500). Fluorophore-conjugated secondary antibodies were purchased from Jackson Labs.

Microscope images were acquired on a DeltaVision Core system (Applied Precision) using an inverted Olympus IX-71 stand, with an Olympus UPlanSApo $\times 100$ oil immersion objective (numerical aperture (NA) 1.4) and a LED light source. Camera (Photometrics Cool Snap HQ), shutter and stage were controlled through SoftWorx (Applied Precision). Z-series were collected with a spacing of $0.2 \mu \mathrm{m}$, and image stacks were subsequently deconvolved in SoftWorx. Immunofluorescence signals in deconvolved images were analysed using ImageJ software (National Institutes of Health, Bethesda, MD). For HEC1 signal quantification, a custom-made macro in Image J modified from (Bodor et al. 2014) was used. Intercentromeric distances were measured with ImageJ using multiple z-stack images. The distances between individual kinetochore pairs that were clearly identified in individual z-stacks were measured.

\section{Immunoblotting analysis}

Whole-cell extracts were prepared from HeLa cells transfected with control siRNA, SMC2 siRNA and the indicated vector DNAs. Immunoblotting analysis was performed using the following primary antibodies: rabbit anti-SMC (WCE 1:500) and mouse anti-Tubulin (Sigma B512, 1:10,000). For protein detection and quantification, we used donkey anti-mouse and antirabbit fluorescence secondary antibodies (LI-COR Bioscience 1:10,000).

\section{Results}

PREditOR (protein reading and editing of residues) effectively removes heterochromatin from pericentromeric regions

To manipulate the epigenetic status of defined chromatin classes, we designed a novel synthetic biology 
approach that allows us to tether chromatin EDITORS to specific regions of the genome, protein reading and editing of residues (PREditOR). PREditOR is based on the use of fusion proteins consisting of three domains (Supplementary Figure 1a): (i) a READER domain that recognizes specific epigenetic modifications, (ii) a fluorescent marker to follow the localization of the fusion protein and (iii) a chromatin EDITOR that functions specifically at or near the tethering site. In order to analyse the role of pericetromeric heterochromatin on chromosome segregation, we fused the $\mathrm{N}$-terminal chromodomain of H3K9-specific methyltransferase SUV39H1 (SUV39H1 $\triangle$ SET) (a READER of H3K9me3) to an EYFP marker (Fig. 1a, b). Removal of the SET domain ensures that this molecule functions solely as a READER and not as an enzymatically active EDITOR.

Immunofluorescence analysis after expression of the SUV39H1 $\triangle$ SET-EYFP fusion protein in HeLa cells showed colocalization with H3K9me3 and CENP-B foci (Fig. 1d and Supplementary Figure 1b). Thus, this fusion protein targets specifically to pericentromeric heterochromatin. SUV39H1 $\Delta$ SET-EYFP is released from chromatin in early mitosis and rebinds later in anaphase (Supplementary Figure 1c). This is most likely due to a methyl/phos switch effect caused by phosphorylation of histone $\mathrm{H} 3$ on Serine 10 catalysed by Aurora B kinase (Fischle et al. 2005; Hirota et al. 2005).

As an EDITOR to remove $\mathrm{H} 3 \mathrm{~K} 9 \mathrm{me} 3$ from pericentromeric regions, we fused SUV39H1 $\Delta$ SETEYFP to the H3K9me3-specific demethylase JMJD2D/KDM4 ( SUV39H1 $\triangle$ SET-EYFP$\mathrm{JMJD}_{2 \mathrm{D}}{ }^{\mathrm{WT}}$ ) (Fig. 1b, c). Two control molecules were also constructed (Fig. 1b, c). The first was a catalytically dead mutant of JMJD2D carrying a mutation in its jmjCenzymatic domain fused to SUV39H1 $\triangle$ SET-EYFP $\left(\mathrm{SUV} 39 H 1 \Delta\right.$ SET-EYFP-JMJD2D $\left.{ }^{\mathrm{D} 195 \mathrm{~A}}\right)$. This molecule targets to heterochromatin but cannot demethylate H3K9. The second was a binding-deficient mutant of SUV39H1 $\triangle$ SET bearing two mutations of its chromatin-binding domain fused to wild type JMJD2D $\left(\mathrm{SUV} 39 \mathrm{H} 1 \Delta \mathrm{SET}^{\mathrm{W} 61 \mathrm{AY} 67 \mathrm{~A}}\right.$-EYFP-JMJD2D ${ }^{\mathrm{WT}}$ ). This molecule has an active demethylase but cannot target specifically to heterochromatin.

Transient expression of SUV39H1 $\Delta$ SET-EYFPJMJD2D ${ }^{\text {WT }}$ in HeLa cells for 48 h efficiently removed H3K9me3 from pericentromeric loci. Immunofluorescence analysis revealed significantly decreased levels of H3K9me3 levels in cells expressing SUV39H1 $\Delta$ SETEYFP-JMJD2D ${ }^{\mathrm{WT}}$ compared to the transfection and tethering controls (EYFP and SUV39H1 $\triangle$ SET-EYFP, respectively) (Fig. 1d, e). Importantly, no differences in $\mathrm{H} 3 \mathrm{~K} 9 \mathrm{me} 3$ levels were observed after expressing either the catalytically dead mutant (SUV39H1 $\triangle$ SET-EYFPJMJD2D ${ }^{\mathrm{D} 195 \mathrm{~A}}$ ) or the binding-deficient mutant (SUV39H1 $\Delta$ SET $^{\mathrm{W} 61 \text { AY67A-EYFP-JMJD2D }}{ }^{\mathrm{WT}}$ ) (Fig. 1d, e). Apparently, JMJD2D only efficiently demethylates $\mathrm{H} 3 \mathrm{~K} 9 \mathrm{me} 3$ when it is tethered to heterochromatic regions. Consistent with these results, immunofluorescence staining for $\operatorname{HP} 1 \alpha$, another hallmark of heterochromatin, revealed a strongly significant decrease in HP $1 \alpha$ foci in cells expressing SUV39H1 $\Delta$ SET-EYFP-JMJD2D ${ }^{\text {WT }}$ compared with cells expressing the other control constructs (Supplementary Figure 1d and e).

We also investigated whether chromosomes overall looked more decondensed after expression of SUV39H1 $\triangle$ SET-EYFP-JMJD2D ${ }^{\text {WT }}$ fusion protein. Although there did appear to be some slight decompaction in live images, when chromosomes were fixed and spreads prepared, no significant differences were seen.

We conclude that PREditOR can effectively remove H3K9me3 and specifically disrupt heterochromatin, releasing downstream heterochromatin READERS such as HP1 $\alpha$. Importantly, JMJD2D only removes heterochromatin when it is tethered to the pericentromeric regions of chromosomes.

Heterochromatin removal causes a mitotic accumulation and chromosome segregation defects

To analyse the effects of heterochromatin removal on cell division, we expressed the different SUV39H1 $\triangle$ SET-EYFP fusion proteins in HeLa cells for $48 \mathrm{~h}$ and examined their effects on mitosis. Our results show a threefold increase in the mitotic index of cells expressing SUV39H1 $\Delta$ SET-EYFP-JMJD2D ${ }^{\text {WT }}$ compared to cells expressing the control fusion proteins (Fig. 2a). The control results demonstrate that SUV39H1 $\triangle$ SET-EYFP binding to pericentromeric regions does not interfere with mitotic progression and that the increase in mitotic index is due to the demethylase activity of JMJD2D.

We observed significantly decreased levels of prophase, metaphase and anaphase cells expressing SUV39H1 $\triangle$ SET-EYFP-JMJD2D ${ }^{\text {WT }}$ compared to controls (Fig. 2b). No difference was observed in the 
frequency of cells in telophase, though a small increase was seen for cells in cytokinesis.

In order to analyse the effects of heterochromatin removal on chromosome segregation, we quantified the frequencies of mitotic abnormalities in HeLa cells expressing the different SUV39H1 $\triangle$ SET-EYFP fusion proteins. We quantified the frequencies of anaphase bridges, lagging chromosomes, uncongressed chromosomes in metaphase and malformed spindles. Overall, cells expressing SUV39H1 $\Delta$ SET-EYFP-JMJD2D ${ }^{\mathrm{WT}}$ showed a significantly increased frequency of abnormal mitosis compared to cells expressing the other vectors (40 vs 8 $15 \%$, respectively) (Fig. 2c, d). In particular, we observed significantly increased frequencies of lagging chromosomes and bridges in cells expressing SUV39H1 $\Delta$ SETEYFP-JMJD2D $^{\mathrm{WT}}$ (Fig. 2e). Although there was no significant increase in multipolar spindles as judged by pericentrin staining, we did see a high frequency of other spindle malformations (Supplementary Figure 2). Consistent with the increased frequencies of mitotic abnormalities, we also observed significantly increased frequencies of micronuclei, a sensitive reporter for chromosome segregation defects, in interphase cells expressing SUV39H1 $\triangle$ SET-EYFP-JMJD2D ${ }^{\text {WT }}$ compared with controls (13 vs 4-6\%) (Fig. 2f, g).

These data suggest that heterochromatin is necessary for correct chromosome segregation during mitosis and that its removal interferes with mitotic progression and chromosome segregation fidelity.

Perturbing heterochromatin leads to centromere defects

Centromeres direct the assembly the kinetochore, a multi-protein complex that binds to microtubules and directs chromosome segregation (Fukagawa and Earnshaw 2014). However, some kinetochore proteins, including the Mis12 complex, have been reported to bind to the heterochromatin flanking the core centrochromatin (Obuse et al. 2004). In view of the chromosome segregation defects reported above, we asked whether heterochromatin removal is associated with kinetochore defects.

Immunofluorescence staining for the outer kinetochore protein HEC1 was performed after expression of the different SUV39H1 $\triangle$ SET-EYFP fusion proteins for $48 \mathrm{~h}$ (the time point at which we observed significant defects on chromosome segregation). We observed mild but significant decreases in levels of HEC1 in cells expressing all of the SUV39H1 $\triangle$ SET-EYFP vectors compared to the transfection control (Fig. 3a, b). This suggests that the binding of SUV39H1 $\triangle$ SET-EYFP alone has an effect on kinetochore structure.

Although all constructs showed statistically significant decreased levels of HEC1 compared with cells expressing EYFP, the greatest decrease was observed in cells expressing SUV39H1 $\triangle$ SET-EYFP-JMJD2D ${ }^{\mathrm{WT}}$ (-49\%). Lesser decreases were observed in cells expressing SUV39H1 $\Delta$ SET-EYFP $(-36 \%)$, SUV39H1 $\Delta$ SET-

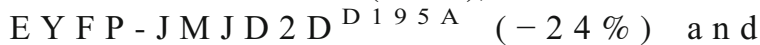
SUV39H1 $\Delta$ SET $^{\text {W61AY67A }}$-EYFP-JMJD2D ${ }^{\text {WT }}(-9 \%)$. Therefore, perturbing heterochromatin has a deleterious effect on kinetochore structure. The SUV39H1 $\Delta$ SET module may exert a dominant-negative effect by competing with READERS that bind to $\mathrm{H} 3 \mathrm{~K} 9 \mathrm{me} 3$. This is consistent with the observation that the SUV39H1 $\Delta$ SET binding mutant exhibited the mildest phenotype.

Pericentromeric heterochromatin has been associated with the maintenance of cohesin in metaphase (Nonaka et al. 2002). After prophase, cohesin complexes are removed from the chromosome arms, but are retained at centromeres as a result of the activity of Shugoshin 1 (SGO1) (Losada et al. 2002). Given previous links between heterochromatin and cohesin in $S$. pombe (Nonaka et al. 2002), we analysed the localization of SGO1 after expressing the different SUV39H1 $\Delta$ SETEYFP fusion proteins in HeLa cells. In transfection controls, SGO1 showed a clear centromeric localization in $95 \%$ of the cells (Fig. 3c, d). Expression of the different SUV39H1 $\Delta$ SET-EYFP proteins resulted in significant increases in the frequency of cells with SGO1 dispersed on chromosome arms (Fig. 3c, d). Thus, SUV39H1 $\Delta$ SET-EYFP binding to pericentromeric heterochromatin perturbs SGO1 centromeric localization. As was the case for HEC1 staining, cells expressing SUV39H1 $\Delta$ SET-EYFP-JMJD2D ${ }^{\text {WT }}$ more frequently exhibited SGO1 localization defects than did cells expressing other SUV39H1 $\Delta$ SET-EYFP controls (Fig. 3c, d).

We conclude that SUV39H1 $\Delta$ SET-EYFP fusion proteins binding to pericentromeres generate mild defects on the kinetochore and SGO1. However, these defects are consistently higher after removing heterochromatin.

Heterochromatin cooperates with condensin to maintain centromeric stiffness

We and others previously showed that the condensin complex is important for maintaining the rigidity of the 


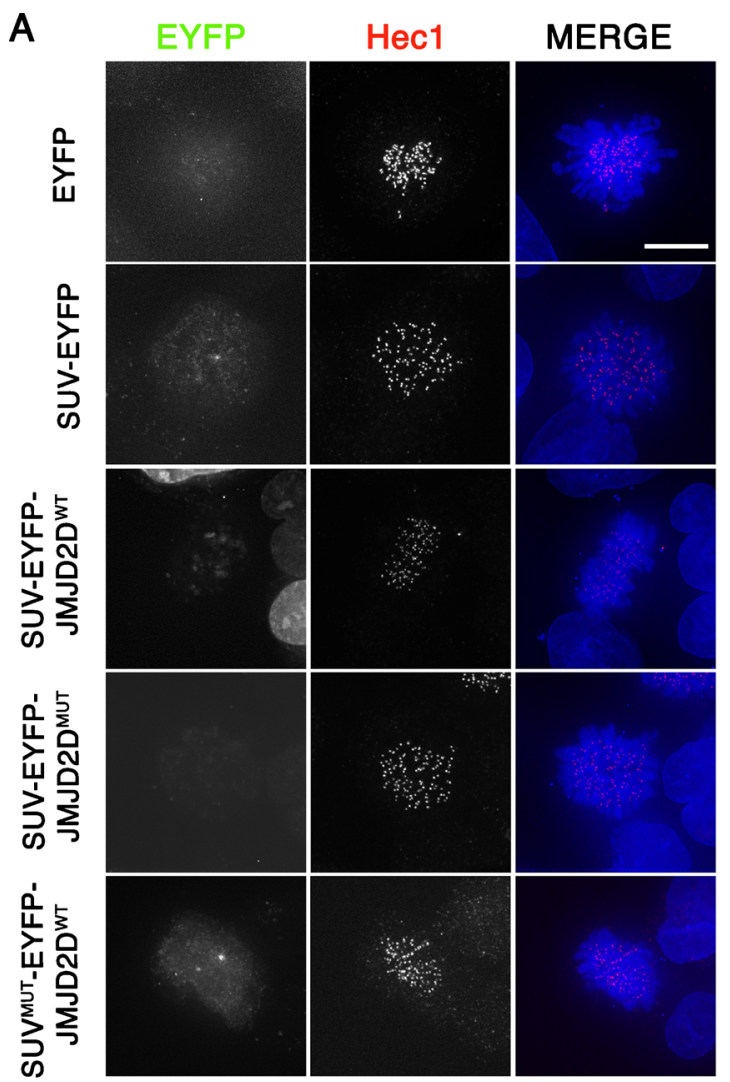
B

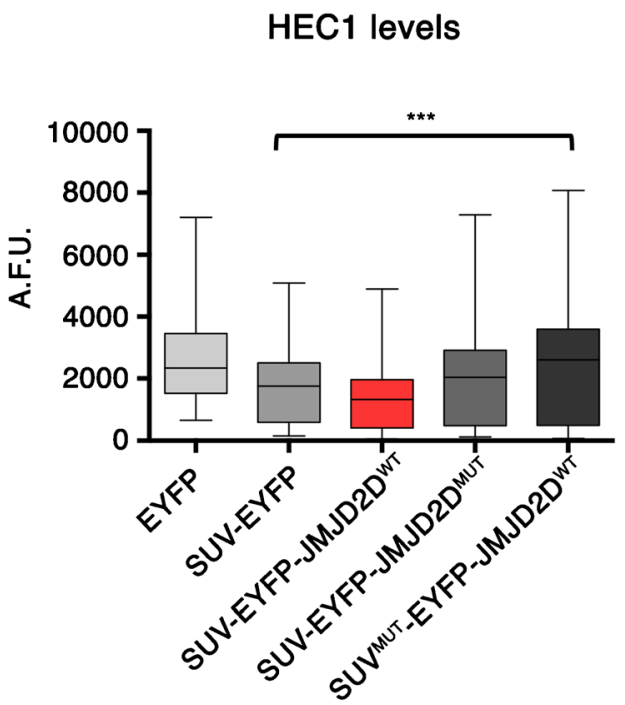

D
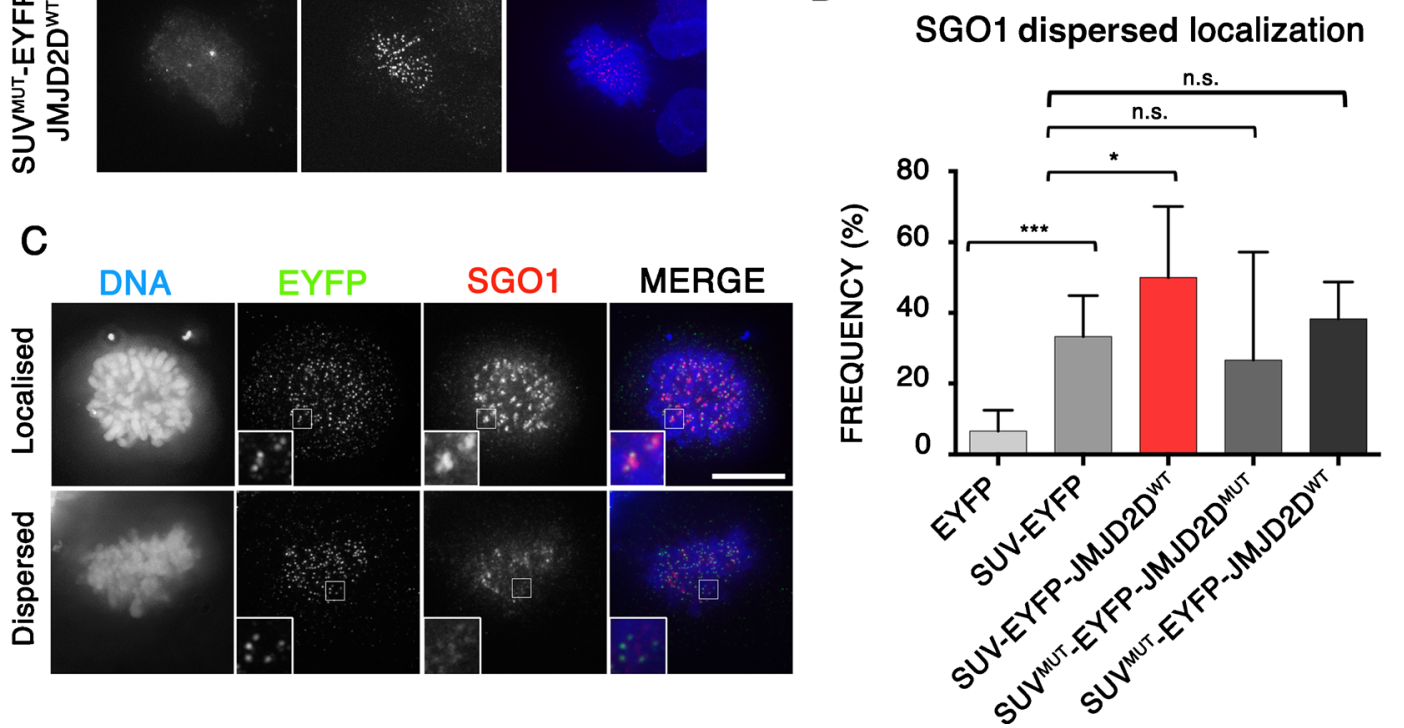

Fig. 3 Heterochromatin removal leads to centromere defects. a Representative immunofluorescence images of HeLa cells expressing the indicated SUV39H1 $\triangle$ SET-EYFP fusion proteins and stained for HEC1. Scale bar $10 \mu \mathrm{m}$. b Quantification of fluorescence signals of HEC1 staining in individual cells transfected as in (a) plotted as arbitrary fluorescence units (A.F.U). Solid bars indicate the medians of two independent experiments and error bars represent the standard error of the

centromere (Gerlich et al. 2006; Ribeiro et al. 2009; Jaqaman et al. 2010). We hypothesized that condensin might act by regulating the compliance of centromeric heterochromatin (Ribeiro et al. 2009). To test the effect mean (s.e.m). c Representative immunofluorescence images showing prometaphase cells with localized (top) or dispersed (bottom) SGO1, using CENP-A as centromere marker. Scale bar $10 \mu \mathrm{m}$. d Analysis of the frequency of cells showing localized or dispersed SGO1 staining after expressing the indicated SUV39H1 $\Delta$ SET-EYFP fusion proteins. Data represent the mean and standard error of the mean (s.e.m) of three independent experiments

of removing heterochromatin on centromere stiffness, we expressed the various SUV39H1 $\Delta$ SET-EYFP fusion proteins in HeLa cells for $48 \mathrm{~h}$ and analysed the distances between sister kinetochores on metaphase 
chromosomes. We observed a significant increase in this distance after expressing SUV39H1 $\Delta$ SET-EYFPJMJD2D ${ }^{\text {WT }}$ compared with controls (Fig. 4a, b). This supports the notion that pericentromeric heterochromatin has a role in maintaining centromeric stiffness.

In order to investigate our hypothesis that there is an interaction between condensin and heterochromatin in maintaining centromeric stiffness, we partly depleted SMC2 in HeLa cells using published siRNAs (Gerlich et al. 2006). Western blot analysis showed a $61 \%$ decrease in SMC2 levels after siRNA transfection (Supplementary Figure 3a). This was confirmed by immunofluorescence analysis, which showed a reduction of SMC2 levels on chromosomes compared with the control siRNA (Supplementary Figure 3b). Although $39 \%$ of the SMC2 remained in cells under these conditions, we observed the characteristic phenotypes of condensin-depleted cells, including dramatic changes in chromosome morphology, increased frequencies of lagging chromosomes and chromosome bridges (Supplementary Figure $3 \mathrm{~b}$ and $\mathrm{c}$ ).

Once the conditions for SMC2 depletion with siRNA were established, we analysed the intercentromeric distances of metaphase chromosomes after expressing either SUV39H1 $\Delta$ SET-EYFP or SUV39H1 $\Delta$ SETEYFP-JMJD2D ${ }^{\mathrm{WT}}$ in the presence or absence of SMC2 depletion (Fig. 4c). Consistent with previous results from our group (Ribeiro et al. 2009), we observed a strong increase in intercentromeric distances in cells depleted of SMC2 compared with those transfected with the control siRNA (Fig. 4d, e). Strikingly, our analysis showed further significant increases of intercentromeric distances in cells expressing SUV39H1 $\triangle$ SET-EYFP-JMJD2D ${ }^{\text {WT }}$ compared with controls expressing SUV39H1 $\Delta$ SET-EYFP. This additional effect upon removal of heterochromatin was seen both in the presence and absence of SMC2 (Fig. 4d, e).

These results show that heterochromatin cooperates with condensin to maintain centromeric stiffness. However, the additive nature of the observed effect suggests that condensin and heterochromatin make at least partly independent contributions.

Heterochromatin is essential for proper chromosome passenger complex localization

The chromosome passenger complex (CPC) of Survivin, INCENP, Borealin and its catalytic subunit Aurora B Kinase localizes to different targets during mitosis, where it regulates key mitotic events (Carmena et al. 2012). In early mitosis, the CPC is localized at inner centromeres, where it ensures that kinetochore-microtubule attachments are correct and regulates the spindle assembly checkpoint. During anaphase, it transfers to the midzone where it regulates the completion of cytokinesis (Fig. 5a) (Carmena et al. 2012).

It has been reported that centromeric HP1 targets the CPC to centromeres in early mitosis (Ainsztein et al. 1998; Liu et al. 2014). In order to study the role of heterochromatin on CPC localization at centromeres, we expressed the different SUV39H1 $\Delta$ SET-EYFP vectors in HeLa cells for $48 \mathrm{~h}$ and analysed the localization of the CPC by staining for Survivin (Fig. 5a, b). In control cells expressing SUV39H1 $\triangle$ SET-EYFP, the $\mathrm{CPC}$ concentrates at centromeres during prometaphase (Fig. 5a, c). Strikingly, our immunofluorescence analysis of cells expressing SUV39H1 $\Delta$ SET-EYFPJMJD2D ${ }^{\text {WT }}$ showed an increased frequency of cells with the CPC dispersed on the chromosome arms in early mitosis (Fig. 5b, c). Moreover, we observed defects in CPC transfer to the midzone in late mitosis (Fig. 5a, b, bottom panels). Expression of SUV39H1 $\triangle$ SET-EYFP-JMJD2D ${ }^{\text {WT }}$ led to an increased frequency of cells in late mitosis in which the CPC remained attached to chromosomes and failed to concentrate at the spindle midzone.

We conclude that heterochromatin is necessary for efficient CPC localization at centromeres and also for its transfer to the midzone in late mitosis.

\section{Discussion}

The ever-expanding panoply of histone modifications function by influencing the overall structure of chromatin and by regulating the binding of chromatin READERS (Bannister and Kouzarides 2011). Epigenetics can be thought of as functioning through EDITOR $\rightarrow$ MARK $\rightarrow$ READER $\rightarrow$ Chromatin State $(\mathrm{E} \rightarrow \mathrm{M} \rightarrow \mathrm{R} \rightarrow \mathrm{C}$ ) pathways that can be artificially engineered by our synthetic biology approach PREditOR. Three examples amongst the many READERS that bind to specific histone modifications include the chromatin remodeller CHD1, which binds to $\mathrm{H} 3 \mathrm{~K} 4 \mathrm{me} 2 / 3$ (Sims et al. 2005), the polycomb repressor complex subunit PRC1, which binds to H3K27me3 (Cao et al. 2002; Levine et al. 2002) and the H3K9 methyltransferase SUV39H1, 
which binds to H3K9me2/3 (Rea et al. 2000). Our PREditOR approach can be applied to study any of these pathways as we have shown in several previous studies focused on the epigenetic landscape of the centromere of a synthetic human artificial chromosome (HAC (Nakano et al. 2008; Cardinale et al. 2009; Bergmann et al. 2011; Martins et al. 2016; Molina et al., 2016).

Here, we describe the first use of PREditOR to study native human chromosomes. We designed a specific PREditOR strategy to tether an EDITOR to constitutive heterochromatin regions as a fusion to the chromodomain of the H3K9-specific methyltransferase SUV39H1 linked to EYFP. It was previously reported that the truncated SUV39H1 protein maintaining only its chromodomain targets specifically to pericentromeric regions (Melcher et al. 2000; Krouwels et al. 2005), which comprise the bulk of constitutive heterochromatin in eukaryotic cells. Consistent with those reports, we observed a strong colocalization of SUV39H1 $\triangle$ SET-EYFP with heterochromatin foci containing the centromeric protein CENP-B in HeLa cells. We also observed a similar colocalization after expressing SUV39H1 $\Delta$ SET-EYFP in other human cell lines, including HT1080 and RPE1hTERT (data not shown). Immunofluorescence analysis staining for $\mathrm{H} 3 \mathrm{~K} 9 \mathrm{me} 3$ and $\mathrm{HP} 1 \alpha$ after expressing SUV39H1 $\triangle$ SET-EYFP-JMJD2D ${ }^{\text {WT }}$ showed residual diffuse antibody signals in all nuclei, but a loss of the characteristic heterochromatin foci (Fig. 1d and Supplementary Figure 1d). These results suggest that SUV39H1 $\Delta$ SET-EYFP expression disrupts pericentromeric heterochromatin.

Removal of heterochromatin leads to defects in mitosis subsequent to delays in prometaphase. Many of these may be explained by defects in localization of the CPC observed after heterochromatin removal (Fig. 5). The CPC controls the proper attachment of kinetochores to microtubules and activates the spindle assembly checkpoint until chromosomes are properly aligned (Carmena et al., 2012).

Importantly, cells expressing several control SUV39H1 $\triangle$ SET-EYFP fusion proteins behaved like the transfection control (EYFP alone). Thus, SUV39H1 $\triangle$ SET binding did not interfere with normal mitotic progression. In contrast, other authors have observed a mitotic arrest after inhibiting SUV39H1 by its specific inhibitor chaetonin, which decreases the levels of heterochromatin (Chu et al. 2014; Chu et al. 2012). This stronger phenotype could be due to off- target effects of the drug or to the action of SUV39H1 on other targets required for mitotic progression.

Disruption of pericentromeric heterochromatin has been reported to affect chromosome segregation and to increase genomic instability in yeast (Ekwall et al. 1995), mice (Peters et al. 2001) and human cells (Slee et al. 2012). Surprisingly, although double null mutants for Suv39h1 and Suv39h2 in mice show severely impaired viability, a low level of live offspring (33\%) was obtained. These double null or Suv39h1-null mice showed an increased predisposition to B cell lymphomas with hyperdiploid karyotypes, suggesting that lack of pericentromeric heterochromatin might increase genomic instability by impairing chromosome segregation (Peters et al. 2001). Consistent with these results, our data show a threefold increase on chromosome segregation defects after removing heterochromatin by expressing SUV39H1 $\Delta$ SET-EYFP-JMJD2D ${ }^{\mathrm{WT}}$ in human cells for $48 \mathrm{~h}$. Since HeLa cells divide approximately once every $24 \mathrm{~h}$, chromosome segregation defects must occur in the first or at most the second mitosis after heterochromatin removal.

Heterochromatin removal appeared to affect several protein complexes that are important for centromere function. Two of these effects were expected. First, we observed that heterochromatin depletion causes an increase in the compliance (stretchiness) of inner centromeres, with the result that the distance between sister kinetochores is increased. This is consistent with previous suggestions that condensin regulates centromeric

Fig. 4 Heterochromatin is necessary to maintain the stiffness of the centromere in metaphase. a Representative immunofluorescence images of HeLa cells expressing the indicated SUV39H1 $\triangle$ SET-EYFP fusion proteins and stained for CENP-C and Tubulin. b Quantification of intercentromeric distances in chromosomes under tension after expressing the indicated SUV39H1 $\triangle$ SET-EYFP fusion proteins. Data represent the mean and standard error of the mean (s.e.m) of three independent experiments. c Immunoblot of whole HeLa cell protein extract transfected with the indicated siRNA and DNAs. Immunoblot for SMC2 with Tubulin as a loading control. d Representative immunofluorescence images of HeLa cells expressing the indicated SUV39H1 $\triangle$ SET-EYFP fusion proteins and transfected with the indicated siRNA. e Quantification of intercentromeric distances in chromosomes under tension after expressing the indicated SUV39H1 $\Delta$ SET-EYFP fusion proteins and siRNAs. Data represent the mean and standard error of the mean (s.e.m) of three independent experiments. Asterisks indicate statistical significant differences compared to EYFP $(* P<0.05$, $* * P<0.01$, $* * * P<0.001 ;$ Student's $t$ test) 
A

EYFP
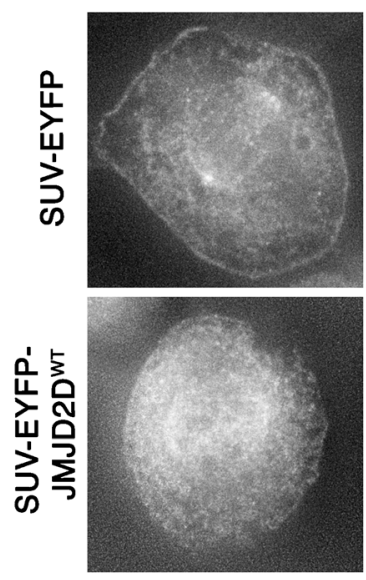

C

Scrambled siRNA SMC2 siRNA

$\begin{array}{cccc}\text { Suv39- } & \text { Suv39-EYFP- } & \text { Suv39- } & \text { Suv39-EYFP } \\ \text { EYFP } & \text { JMJD2D } & \text { EYFP } & \text { JMJD2D }\end{array}$

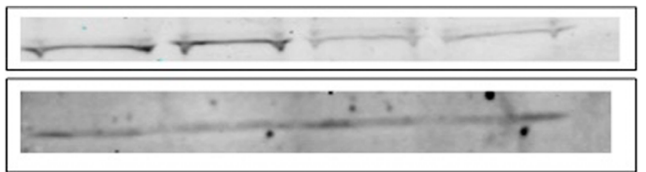

SMC2

Tubulin

D

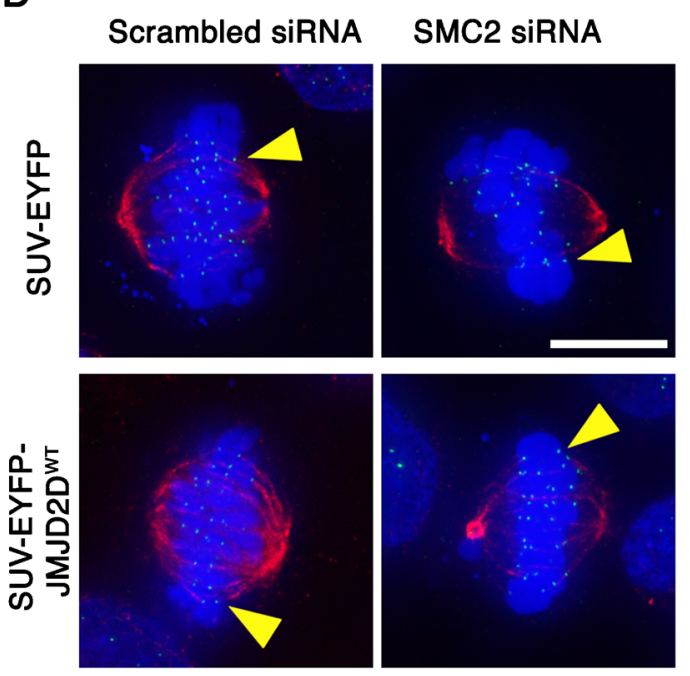

B

Intercentromeric distance

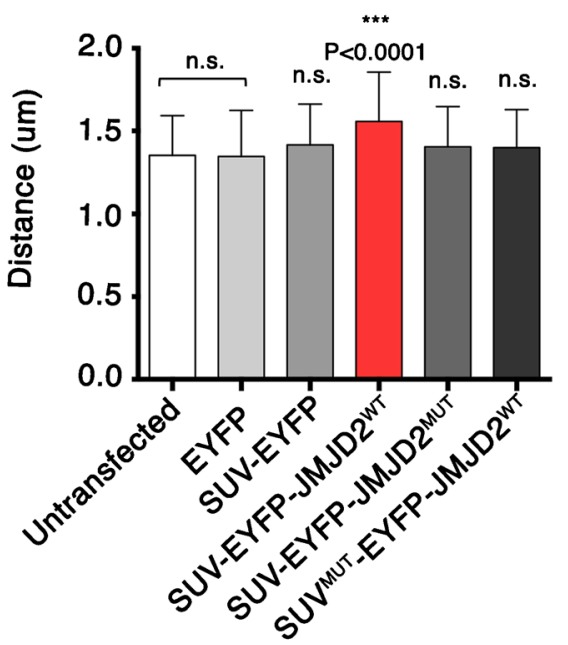

E

Intercentromeric distance

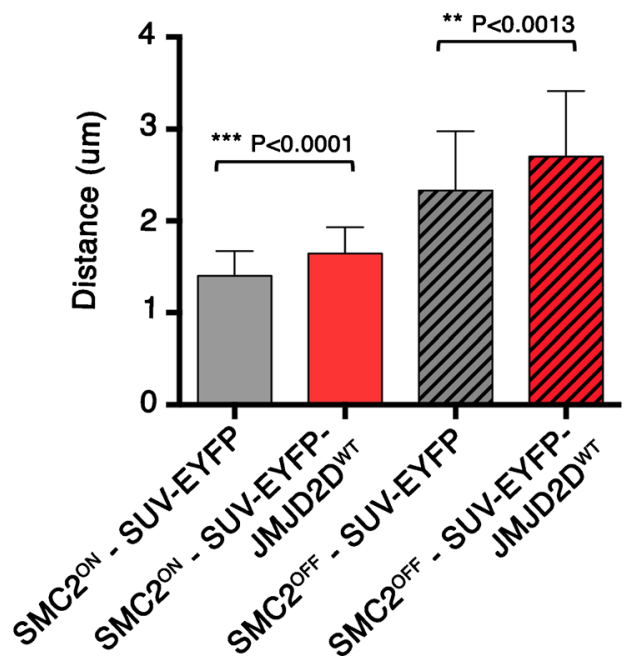

stiffness by regulating the compliance of centromeric heterochromatin (Ribeiro et al. 2009; Jaqaman et al.
2010). It is not clear if this effect is due to changes in the higher-order packing of the chromatin fibre or is 
A

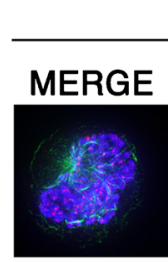

SUV39-EYFP
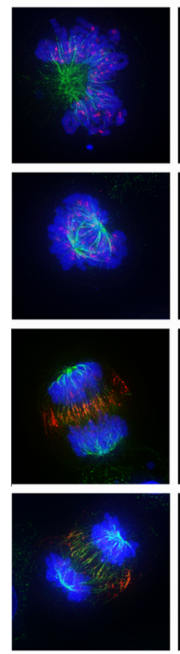

C

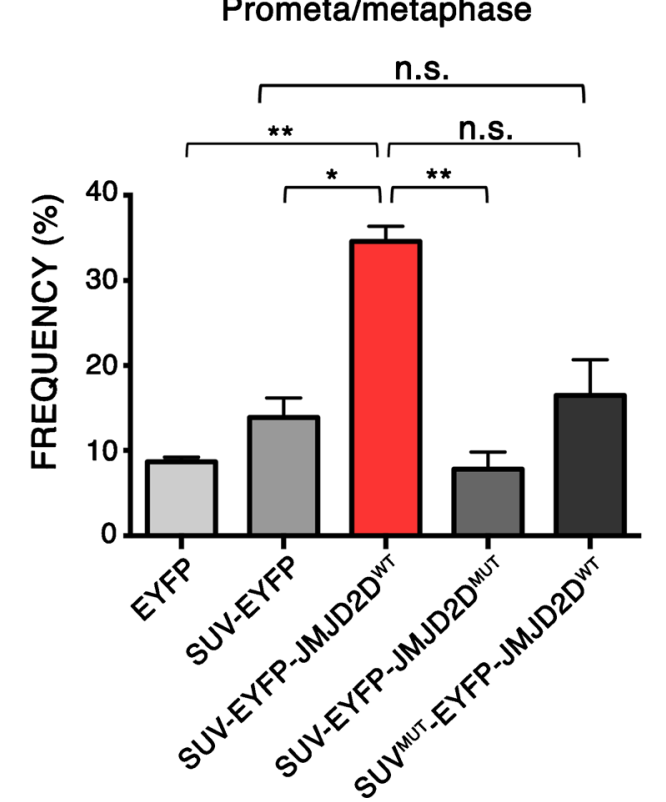

Fig. 5 Heterochromatin removal disrupts chromosomal passenger localization in mitosis. a, b Representative immunofluorescence images of HeLa cells expressing SUV39H1 $\triangle$ SET-EYFP (a) or SUV39H1 $\triangle$ SET-EYFP-JMJD2D ${ }^{\text {WT }}$ (b) fusion protein and stained for Survivin and Tubulin. Scale bar $10 \mu \mathrm{m}$. c Analysis of the frequency of cells showing dispersed $\mathrm{CPC}$ in prometaphase
Dispersed CPC Prometa/metaphase
B

SUV39-EYFP-JMJD2D ${ }^{\text {wT }}$

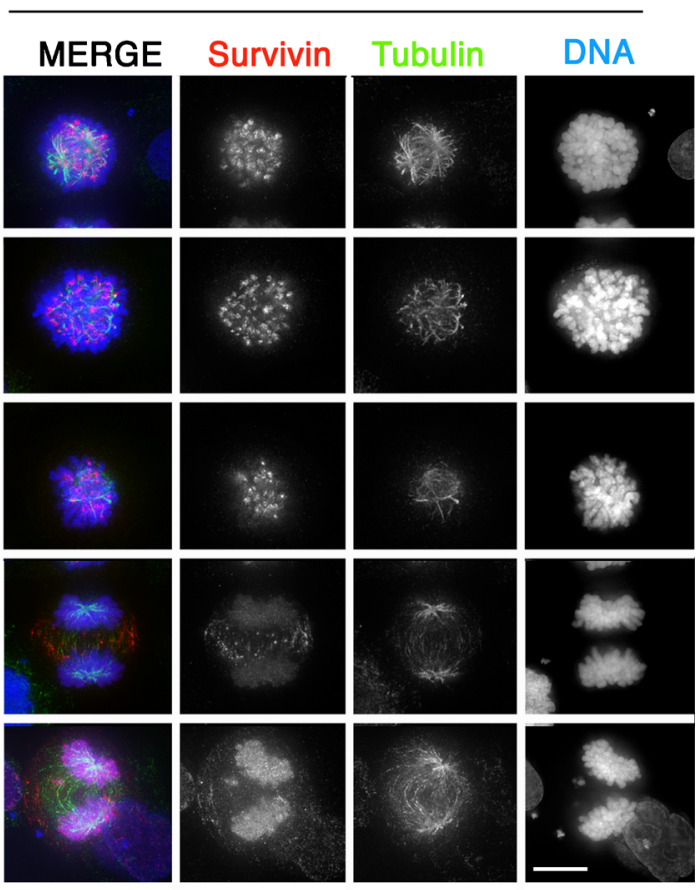
(1)

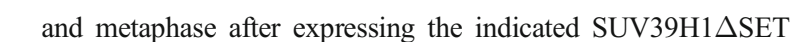
fusion proteins. Data represent the mean and standard error of the mean (s.e.m) of two independent experiments. Asterisks indicate statistical significant differences compared to EYFP (*P $P<0.05, * * P<0.01 ;$ Student's $t$ test) 
mediated by centromeric cohesin complexes, as heterochromatin removal resulted in SGO1 displacement, which might result in lower levels of cohesin at the centromere. The association of heterochromatin with cohesin is currently a matter of debate (Bernard et al. 2001; Koch et al. 2008). Since SGO1 is involved in cohesin complex maintenance at centromeres after prophase until anaphase onset, our results suggest that interfering with SGO1 targeting to centromeres caused by heterochromatin removal might perturb sister chromatid cohesion.

We also observed that centromeric heterochromatin is required for efficient localization of the chromosomal passenger complex (CPC) to inner centromeres during early mitosis. This could be predicted from results indicating that binding to SGO1 (Yamagishi et al. 2010) and to heterochromatin protein HP $1 \alpha$ are both important for targeting of the CPC in early mitosis. As stated above, defects in CPC localization may explain many of the mitotic defects observed following heterochromatin removal.

Binding of SUV39H1 $\Delta$ SET fusion proteins to pericentromeric heterochromatin had a small, but reproducible effect on assembly of the kinetochore. This was not expected, because the kinetochore assembles on the surface of CENP-A-containing centrochromatin and not on the pericentromere. Indeed, in preliminary results, we noticed an increase in the amount of CENP-A associated with centromeres when heterochromatin was depleted. This is consistent with previous results in which the $\mathrm{Rb}$ pathway was perturbed, leading to a decrease in H3K9 methylation (Sullivan et al. 2011). SUV39H1 has a dual role in heterochromatin formation and maintenance: it acts as a methyltransferase and also has a structural role at pericentromeric heterochromatin by binding HP $1 \alpha$ (Haldar et al. 2011). Overexpressed SUV39H1 $\Delta$ SET might affect kinetochore assembly either by perturbing HP1 $\alpha$ dynamics, or possibly as a result of its effects on SGO1 or CPC localization.

The present results demonstrate the utility of the PREditOR approach for epigenetic engineering - in this case to specifically remove heterochromatin from dividing cells. It had been widely assumed that heterochromatin would be important for mitotic chromosome segregation, but previous studies were plagued by the possibility of off-target effects of drugs and genetic manipulations. Here, we confirm that heterochromatin is indeed important for mitotic chromosome segregation, although the effects of heterochromatin removal on pericentromeric heterochromatin structure, mitotic progression and on chromosome segregation are remarkably mild. We conclude that heterochromatin at centromeres is necessary to maintain genomic stability.

Acknowledgements We thank Nuno Martins for interesting discussions that contributed to the development of the PREditOR approach. O.M. was funded by the European Molecular Biology Organization (long-term EMBO fellowship; ALTF-453-2012). This work was funded by the Wellcome Trust, of which W.C.E. is a Principal Research Fellow (grant number 073915). The Wellcome Trust Centre for Cell Biology is supported by core grant numbers 077707 and 092076.

\section{Compliance with ethical standards}

Conflict of Interest The authors declare that they have no conflict of interest.

Open Access This article is distributed under the terms of the Creative Commons Attribution 4.0 International License (http:// creativecommons.org/licenses/by/4.0/), which permits unrestricted use, distribution, and reproduction in any medium, provided you give appropriate credit to the original author(s) and the source, provide a link to the Creative Commons license, and indicate if changes were made.

\section{References}

Ainsztein AM, Kandels-Lewis SE, Mackay AM, Earnshaw WC (1998) INCENP centromere and spindle targeting: identification of essential conserved motifs and involvement of heterochromatin protein HP1. J Cell Biol 143:1763-1774

Aldrup-Macdonald ME, Sullivan BA (2014) The past, present, and future of human centromere genomics. Genes 5:33-50

Allshire RC, Nimmo ER, Ekwall K, Javerzat JP, Cranston G (1995) Mutations derepressing silent centromeric domains in fission yeast disrupt chromosome segregation. Genes Dev 9:218-233

Bannister AJ, Kouzarides T (2011) Regulation of chromatin by histone modifications. Cell Res 21:381-395. doi:10.1038 /cr.2011.22

Bannister AJ, Zegerman P, Partridge JF, Miska EA, Thomas JO, Allshire RC, Kouzarides T (2001) Selective recognition of methylated lysine 9 on histone H3 by the HP1 chromo domain. Nature 410:120-124. doi:10.1038/35065138

Bergmann JH et al (2011) Epigenetic engineering shows $\mathrm{H} 3 \mathrm{~K} 4 \mathrm{me} 2$ is required for HJURP targeting and CENP-A assembly on a synthetic human kinetochore. EMBO J 30: 328-340. doi:10.1038/emboj.2010.329

Bernard P, Maure JF, Partridge JF, Genier S, Javerzat JP, Allshire RC (2001) Requirement of heterochromatin for cohesion at centromeres. Science 294:2539-2542. doi:10.1126 /science. 1064027

Bodor DL et al (2014) The quantitative architecture of centromeric chromatin. eLife 3:e02137. doi:10.7554/eLife.02137 
Cao R et al (2002) Role of histone H3 lysine 27 methylation in polycomb-group silencing. Science 298:1039-1043. doi:10.1126/science.1076997

Cardinale S et al (2009) Hierarchical inactivation of a synthetic human kinetochore by a chromatin modifier. Mol Biol Cell 20:4194-4204. doi:10.1091/mbc.E09-06-0489

Carmena M, Wheelock M, Funabiki H, Earnshaw WC (2012) The chromosomal passenger complex (CPC): from easy rider to the godfather of mitosis. Nat Rev Mol Cell Biol 13:789-803. doi:10.1038/nrm3474

Chu L et al (2014) The spatiotemporal dynamics of chromatin protein HP1alpha is essential for accurate chromosome segregation during cell division. J Biol Chem 289:26249-26262. doi:10.1074/jbc.M114.581504

Chu L et al (2012) SUV39H1 orchestrates temporal dynamics of centromeric methylation essential for faithful chromosome segregation in mitosis. J Mol Cell Biol 4:331-340. doi:10.1093/jmcb/mjs023

Earnshaw WC, Migeon BR (1985) Three related centromere proteins are absent from the inactive centromere of a stable isodicentric chromosome. Chromosoma 92:290-296

Ekwall K, Javerzat JP, Lorentz A, Schmidt H, Cranston G, Allshire R (1995) The chromodomain protein Swi6: a key component at fission yeast centromeres. Science 269:1429-1431

Fischle W et al (2005) Regulation of HP1-chromatin binding by histone $\mathrm{H} 3$ methylation and phosphorylation. Nature 438: 1116-1122. doi:10.1038/nature04219

Folco HD, Pidoux AL, Urano T, Allshire RC (2008) Heterochromatin and RNAi are required to establish CENPA chromatin at centromeres. Science 319:94-97. doi:10.1126 /science. 1150944

Fukagawa T, Earnshaw WC (2014) The centromere: chromatin foundation for the kinetochore machinery. Dev Cell 30:496508. doi:10.1016/j.devcel.2014.08.016

Gartenberg M (2009) Heterochromatin and the cohesion of sister chromatids. Chromosom Res 17:229-238. doi:10.1007 /s10577-008-9012-z

Gerlich D, Hirota T, Koch B, Peters JM, Ellenberg J (2006) Condensin I stabilizes chromosomes mechanically through a dynamic interaction in live cells. Curr Biol 16:333-344. doi:10.1016/j.cub.2005.12.040

Haldar S, Saini A, Nanda JS, Saini S, Singh J (2011) Role of Swi6/ HP1 self-association-mediated recruitment of Clr4/Suv39 in establishment and maintenance of heterochromatin in fission yeast. J Biol Chem 286:9308-9320. doi:10.1074/jbc. M110.143198

Hirota T, Lipp JJ, Toh BH, Peters JM (2005) Histone H3 serine 10 phosphorylation by Aurora B causes HP1 dissociation from heterochromatin. Nature 438:1176-1180. doi:10.1038 /nature 04254

Jaqaman $\mathrm{K}$ et al (2010) Kinetochore alignment within the metaphase plate is regulated by centromere stiffness and microtubule depolymerases. J Cell Biol 188:665-679. doi:10.1083 /jcb.200909005

Koch B, Kueng S, Ruckenbauer C, Wendt KS, Peters JM (2008) The Suv39h-HP1 histone methylation pathway is dispensable for enrichment and protection of cohesin at centromeres in mammalian cells. Chromosoma 117:199-210. doi:10.1007/s00412-007-0139-Z

Krouwels IM, Wiesmeijer K, Abraham TE, Molenaar C, Verwoerd NP, Tanke HJ, Dirks RW (2005) A glue for heterochromatin maintenance: stable SUV39H1 binding to heterochromatin is reinforced by the SET domain. J Cell Biol 170:537-549. doi:10.1083/jcb.200502154

Levine SS, Weiss A, Erdjument-Bromage H, Shao Z, Tempst P, Kingston RE (2002) The core of the polycomb repressive complex is compositionally and functionally conserved in flies and humans. Mol Cell Biol 22:6070-6078

Liu X et al (2014) Chromatin protein HP1alpha interacts with the mitotic regulator Borealin protein and specifies the centromere localization of the chromosomal passenger complex. $\mathrm{J}$ Biol Chem 289:20638-20649. doi:10.1074/jbc. M114.572842

Losada A, Hirano M, Hirano T (2002) Cohesin release is required for sister chromatid resolution, but not for condensinmediated compaction, at the onset of mitosis. Genes Dev 16:3004-3016. doi:10.1101/gad.249202

Martins NM, Bergmann JH, Shono N, Kimura H, Larionov V, Masumoto H, Earnshaw WC (2016) Epigenetic engineering shows that a human centromere resists silencing mediated by H3K27me3/K9me3. Mol Biol Cell 27:177-196. doi:10.1091 /mbc.E15-08-0605

Melcher M, Schmid M, Aagaard L, Selenko P, Laible G, Jenuwein T (2000) Structure-function analysis of SUV39H1 reveals a dominant role in heterochromatin organization, chromosome segregation, and mitotic progression. Mol Cell Biol 20:37283741

Molina O, Vargiu G, Abad MA, Zhiteneva A, Jeyaprakash AA, Masumoto H, Kouprina N, Larionov V, Earnshaw WC (2016) Epigenetic engineering reveals a balance between histone modifications and transcription in kinetochore maintenance. Nat Commun. doi:10.1038/ncomms 13334

Nakano M et al (2008) Inactivation of a human kinetochore by specific targeting of chromatin modifiers. Dev Cell 14:507522. doi:10.1016/j.devcel.2008.02.001

Nonaka N, Kitajima T, Yokobayashi S, Xiao G, Yamamoto M, Grewal SI, Watanabe Y (2002) Recruitment of cohesin to heterochromatic regions by Swi6/HP1 in fission yeast. Nat Cell Biol 4:89-93. doi:10.1038/ncb739

Oberdoerffer P, Sinclair DA (2007) The role of nuclear architecture in genomic instability and ageing. Nat Rev Mol Cell Biol 8:692-702. doi: $10.1038 / \mathrm{nrm} 2238$

Obuse C, Iwasaki O, Kiyomitsu T, Goshima G, Toyoda Y, Yanagida M (2004) A conserved Mis12 centromere complex is linked to heterochromatic HP1 and outer kinetochore protein Zwint-1. Nat Cell Biol 6:1135-1141. doi:10.1038 /ncb1187

Olszak AM et al (2011) Heterochromatin boundaries are hotspots for de novo kinetochore formation. Nat Cell Biol 13:799808. doi:10.1038/ncb2272

Peng JC, Karpen GH (2007) H3K9 methylation and RNA interference regulate nucleolar organization and repeated DNA stability. Nat Cell Biol 9:25-35. doi:10.1038/ncb1514

Peters AH et al (2001) Loss of the Suv39h histone methyltransferases impairs mammalian heterochromatin and genome stability. Cell 107:323-337

Rea S et al (2000) Regulation of chromatin structure by sitespecific histone H3 methyltransferases. Nature 406:593599. doi:10.1038/35020506

Ribeiro SA et al (2009) Condensin regulates the stiffness of vertebrate centromeres. Mol Biol Cell 20:2371-2380. doi:10.1091/mbc.E08-11-1127 
Saksouk N, Simboeck E, Dejardin J (2015) Constitutive heterochromatin formation and transcription in mammals. Epigenetics Chromatin 8:3. doi:10.1186/1756-8935-8-3

Sims RJ 3rd, Chen CF, Santos-Rosa H, Kouzarides T, Patel SS, Reinberg D (2005) Human but not yeast CHD1 binds directly and selectively to histone $\mathrm{H} 3$ methylated at lysine 4 via its tandem chromodomains. J Biol Chem 280:41789-41792. doi:10.1074/jbc.C500395200

Slee RB et al (2012) Cancer-associated alteration of pericentromeric heterochromatin may contribute to chromosome instability. Oncogene 31:3244-3253. doi:10.1038/onc.2011.502

Sullivan BA, Karpen GH (2004) Centromeric chromatin exhibits a histone modification pattern that is distinct from both euchromatin and heterochromatin. Nat Struct Mol Biol 11:10761083. doi:10.1038/nsmb845
Sullivan LL, Boivin CD, Mravinac B, Song IY, Sullivan BA (2011) Genomic size of CENP-A domain is proportional to total alpha satellite array size at human centromeres and expands in cancer cells. Chromosome research: an international journal on the molecular, supramolecular and evolutionary aspects of chromosome biology 19:457-470. doi:10.1007/s10577-011-9208-5

Yamagishi Y, Honda T, Tanno Y (2010) Watanabe Y. Two histone marks establish the inner centromere and chromosome biorientation Science 330:239-243. doi:10.1126 /science.1194498

Yamagishi Y, Sakuno T, Shimura M (2008) Watanabe Y. Heterochromatin links to centromeric protection by recruiting shugoshin Nature 455:251-255. doi:10.1038 /nature 07217 